\title{
PlexinA3 Restricts Spinal Exit Points and Branching of Trunk Motor Nerves in Embryonic Zebrafish
}

\author{
Julia Feldner, ${ }^{2,5}$ Michell M. Reimer, ${ }^{1}$ Jörn Schweitzer, ${ }^{3}$ Björn Wendik, ${ }^{3}$ Dirk Meyer, ${ }^{4}$ Thomas Becker, ${ }^{1,5}$ and \\ Catherina G. Becker ${ }^{1,5}$ \\ ${ }^{1}$ Centre for Neuroscience Research, Royal (Dick) School of Veterinary Studies, University of Edinburgh, Edinburgh EH9 1QH, United Kingdom, ${ }^{2}$ Institute \\ for Molecular Bioscience, University of Queensland, St Lucia QLD 4072, Australia, ${ }^{3}$ Institut für Biologie 1, Universität Freiburg, Freiburg, D-79104, \\ Germany, ${ }^{4}$ Institut für Molekularbiologie, Leopold-Franzens-Universität Innsbruck, A-6020 Innsbruck, Austria, and ${ }^{5}$ Zentrum für Molekulare \\ Neurobiologie, University of Hamburg, D-20246 Hamburg, Germany
}

The pioneering primary motor axons in the zebrafish trunk are guided by multiple cues along their pathways. Plexins are receptor components for semaphorins that influence motor axon growth and path finding. We cloned plexinA3 in zebrafish and localized plexinA3 mRNA in primary motor neurons during axon outgrowth. Antisense morpholino knock-down led to substantial errors in motor axon growth. Errors comprised aberrant branching of primary motor nerves as well as additional exit points of axons from the spinal cord. Excessively branched and supernumerary nerves were found in both ventral and dorsal pathways of motor axons. The trunk environment and several other types of axons, including trigeminal axons, were not detectably affected by plexinA3 knock-down. RNA overexpression rescued all morpholino effects. Synergistic effects of combined morpholino injections indicate interactions of plexinA3 with semaphorin3A homologs. Thus, plexinA3 is a crucial receptor for axon guidance cues in primary motor neurons.

Key words: primary motor neurons; pioneer axons; neuropilin; semaphorin; zebrafish; development

\section{Introduction}

Axonal path finding during development is determined by an array of overlapping pathway cues and receptors. PlexinA1 to A4 are coreceptors for axon-repelling or -attracting class 3 extracellular semaphorins. It is thought that neuropilin-1 (NRP1) or NRP2 are the ligand-binding part, and plexins are the signaltransducing part of semaphorin class 3 receptors (for a recent review, see Kruger et al., 2005). Removing individual components from this guidance network leads to specific defects of nerve growth (Giger et al., 2000; Huber et al., 2005; Yaron et al., 2005), indicating distinct roles for different ligand/receptor combinations in the path finding of different axon populations.

Studying the outgrowth of primary motor axons in zebrafish offers the opportunity to unravel the role of individual guidance cues and receptors at the level of single pioneer axons in vivo. Three primary motor neurons per trunk hemisegment grow axons out of the spinal cord along a common pathway in the middle of each segment up to the horizontal myoseptum. The axon of the caudal primary motor neuron $(\mathrm{CaP})$ is the first to grow, followed by the axons of the middle $(\mathrm{MiP})$ and rostral primary motor neurons (RoP). At the horizontal myoseptum, the CaP axon continues its growth toward the ventral somite, pioneering the ven-

\footnotetext{
Received Dec. 25, 2006; accepted March 30, 2007

This work was supported by the Deutsche Forschungsgemeinschaft and the Royal Society. We thank Dr. Melitta Schachner for support, Dr. Peter Brophy for support and use of his microscope, Laura Sander for correction of the English, and Bénédicte Autin for excellent technical assistance.

Correspondence should be addressed to Catherina G. Becker at the above address. E-mail: catherina.becker@ed.ac.uk.

DOI:10.1523/JNEUROSCI.1132-07.2007

Copyright $\odot 2007$ Society for Neuroscience $\quad$ 0270-6474/07/274978-06\$15.00/0
}

tral motor nerve, whereas the MiP axon retracts and grows toward the dorsal somite. The RoP axon takes a lateral path from the horizontal myoseptum (for review, see Beattie, 2000).

Semaphorin 3A1 (sema3A1) and sema3A2 (zebrafish homologs of mammalian sema3A) are expressed in the trunk environment. Overexpression of either ligand reduces growth of primary motor axons (Roos et al., 1999; Halloran et al., 2000). Antisense morpholino oligonucleotide knock-down of sema3A1 leads mainly to aberrant branching of the CaP axon (Sato-Maeda et al., 2006). Knock-down of NRP1a alone or in double knockdown experiments with semaphorin ligands leads to nerve branching, additional exit points of axons from the spinal cord, and ventral displacement of neuronal somata along the extraspinal motor axon pathway (Feldner et al., 2005). This suggests that semaphorins guide primary motor axons by repellent mechanisms via NRP1a-containing axonal receptors. Although this powerful system has attracted significant attention, for example in forward genetic screens for axon guidance molecules (Birely et al., 2005; Gulati-Leekha and Goldman, 2006), the role of plexins has not been examined. The only class A member of the plexin family characterized in zebrafish so far is plexinA4, which is not expressed in primary trunk motor neurons (Miyashita et al., 2004).

Here, we clone plexinA3 in zebrafish and show by antisense morpholino knock-down that plexinA3 is necessary for unbranched nerve growth and to restrict spinal exit points of primary motor axons to a midsegmental position. Morpholino coinjection experiments suggest that plexinA3 belongs to a receptor complex for semaphorins in primary motor neurons. 


\section{Materials and Methods}

Zebrafish. Zebrafish were bred under standard conditions (Kimmel et al., 1995). The transgenic fish in which the promoter for hb9 drives expression of green fluorescent protein (GFP) in primary motor neurons has been described previously (Flanagan-Steet et al., 2005).

Cloning procedure. A search of the Ensembl database (www. ensembl.org/Danio_rerio/) predicted ENSDARG00000016216 (Ensembl release 19) on zebrafish chromosome 8 to be most closely related to mouse and human plexinA3. The predicted transcript lacked part of the $5^{\prime}$ region, which was included in the expressed sequenced-tagged clone 24185179. Based on this information, the entire gene could be isolated from cDNA prepared from adult zebrafish brains using PCR with the proofreading polymerase PfuUltra (Stratagene, Cambridge, UK). The GenBank accession number for plexinA3 is EF538743.

Injection of mRNA and morpholinos. Two morpholinos of nonoverlapping sequence for plexinA3 (plexinA3 morpholino1, ATACCAGCAGCCACAAGGACCTCAT; plexinA3 morpholino2, AGCTCTTCCCTCAAGCGTATTCCAG) and a morpholino in which five bases were mismatched based on morpholinol (PlexinA3 $5 \mathrm{~mm}$ morpholino, ATACCACCACCCAGAACGACCTGAT) were purchased from Gene Tools (Philomath, OR). Morpholinos against sema3A1, sema3A2 (Feldner et al., 2005), and NRP1a (Lee et al., 2002) have been described previously.

Messenger RNAs for injection experiments were synthesized as described previously (Feldner et al., 2005). Partial sequences of plexinA3 that contained untranslated $5^{\prime}$ sequences followed by a myc-tag were used to determine binding efficiency of the morpholinos. Full-length plexinA3 mRNA followed by a myc-tag was synthesized for rescue experiments. This construct did not contain the recognition sequence of morpholino2.

For injections, rhodamine dextran $\left(0.8 \% ; M_{\mathrm{r}}=10 \times 10^{3}\right.$; Invitrogen, Paisley, UK) was added to mRNA or morpholino solutions. A glass micropipette was filled with the mRNA $(1-2 \mu \mathrm{g} / \mu \mathrm{l})$ or morpholino solutions ( $\leq 2 \mathrm{~mm}$ ), and a volume of 0.5 to $1 \mathrm{nl}$ per egg (one-to-four-cell stage) was injected as described previously (Feldner et al., 2005). All injected animals showed normal overall growth and differentiation of nervous structures, such as head commissures, the dorsoventral diencephalic tract, and peripheral nerves, as indicated by anti-tubulin immunohistochemistry. Development was not retarded by any experimental procedures, as indicated by segmental positions of the lateral line nerve primordium (prim-stage) (Kimmel et al., 1995) that were not altered, compared with uninjected embryos (data not shown). Viability was not compromised by RNA or morpholino injections compared with uninjected embryos.

In situ hybridization. A full-length PlexinA3 probe was labeled with digoxigenin using the Megascript kit (Ambion, Warrington, UK) and used on 16 and $24 \mathrm{~h}$ post fertilization (hpf) whole-mounted embryos as described previously (Feldner et al., 2005).

Immunohistochemistry. Whole-mount immunohistochemistry was performed as described previously (Feldner et al., 2005). Ventral motor axons were labeled with a monoclonal antibody against acetylated tubulin (6-11B-1; Sigma-Aldrich, Poole, UK). GFP was immunodetected after in situ hybridization with a polyclonal antibody (AB3080P; Millipore, Bedford, MA). Different antibodies were used to label trunk structures in morpholino-injected animals (1 mM PlexinA3 morpholino1) (supplemental Fig. 3, available at www.jneurosci.org as supplemental material). The 412 monoclonal antibody to the HNK-1 epitope labels motor axons, as described previously (Becker et al., 2001). Monoclonal antibody $(\mathrm{mAb}) \mathrm{CS}-56$ to chondroitin sulfates labels the spinal floor plate and vertical myosepta (Bernhardt and Schachner, 2000) and was purchased from Sigma-Aldrich. A polyclonal antibody against tenascin-C of zebrafish (Tongiorgi, 1999) is used as a marker of the horizontal myoseptum region (Schweitzer et al., 2005). The 40.2D6 antibody to islet-1/-2 that labels motor neuron somata and Rohon-Beard cells (Feldner et al., 2005) and the antibody $3 \mathrm{~A} 10$ to a neurofilament-associated antigen that labels commissural primary ascending interneurons and Mauthner axons in the spinal cord (Feldner et al., 2005) were both developed by Dr. T. M. Jessell (Columbia University, New York, NY). These antibodies, as well as the 4D9 antibody to engrailed that labels muscle pioneer cells at the horizontal myoseptum (Patel et al., 1989), were obtained as cell culture supernatants from the Developmental Studies Hybridoma Bank maintained by The University of Iowa, Department of Biological Sciences (Iowa city, IA). To reveal potential alterations in the trunk environment in those segments in which nerve growth was aberrant, antitenascin- $\mathrm{C}$ and anti-engrailed immunolabeling was combined with the axonal markers anti-HNK-1 and anti-tubulin, respectively. For each antibody or combination of antibodies, 11-28 embryos were analyzed. Secondary antibodies were purchased from Dianova (Hamburg, Germany).

Analysis of nerve growth. Anti-tubulin immunolabeled peripheral nerves were analyzed in whole-mounted $24 \mathrm{hpf}$ embryos as described previously (Feldner et al., 2005). Briefly, embryos were scored as affected by nerve branching when at least two of the ventral motor nerves were branched at or above the level of the ventral edge of the notochord. Embryos were scored as affected by multiple exits when at least one hemisegment showed more than one exit point of ventral motor axons from the spinal cord. The rostral 12 pairs of motor nerves were examined. Numbers of peripheral trigeminal axons were assessed as described previously (Becker et al., 2002).

To analyze the dorsal motor axon pathway in $31 \mathrm{hpf}$ hb9:GFP transgenic fish, trunk regions at the level of the yolk extension were scanned on a confocal microscope. Seven segments on both sides of the embryos were assessed for more than one nerve present in the dorsal somite and for excessive branching. For the latter, we scored branching of the nerve ventral to the level of the GFP-positive neuronal somata in the spinal cord. Values for affected embryos are given as mean \pm SEM. Statistical analyses were done using Fisher's exact test unless indicated otherwise.

\section{Results}

\section{Cloning of plexinA3 in zebrafish}

Zebrafish plexinA3 was cloned as described in Materials and Methods. The general domain structure of the deduced protein (1892 aa) is identical to that of plexinA3 in other vertebrate species: a semaphorin domain, followed by three Met-related sequence domains, four IPT (immunoglobulin-like fold shared by plexins and transcription factors) motifs, and the characteristic intracellular plexin domain at the $\mathrm{C}$ terminus. The transmembrane domain of the zebrafish protein is located between the IPT motifs and the Plexin domain and comprises amino acids 12411263 (Fig. 1A).

The cloned protein has significant structural homology (supplemental Fig. 1, available at www.jneurosci.org as supplemental material) and overall amino acid identity (73\%) with human (Maestrini et al., 1996) and mouse (Kameyama et al., 1996) plexinA3. In a phylogenetic tree constructed using the Clustal method (Chenna et al., 2003), zebrafish plexinA3 segregated with plexinA3 homologs of other species (Fig. $1 \mathrm{~B}$ ). These data strongly suggest that we cloned a species homolog of plexinA3. A search for a second paralog of plexinA3 using BLAST analysis of the cloned sequence on the zebrafish genome (Ensembl release 43) had a negative result.

\section{PlexinA3 is expressed in primary motor neurons during axon outgrowth}

In situ hybridization indicated expression of plexinA3 mRNA mainly in the developing nervous system but also in the developing heart (data not shown) and the non-neural tissue at the tip of the tail. Conspicuous expression was detected in the telencephalon, epiphysis, tegmentum, and regular cell clusters in the hindbrain at $24 \mathrm{hpf}$, suggesting expression in differentiating neurons. Low-level expression was found in cranial ganglia and the spinal cord (supplemental Fig. $2 A, B$, available at www.jneurosci.org as supplemental material). However, a particularly strong signal was found in regular clusters of cells at the ventral edge of the spinal cord at 16 and $24 \mathrm{hpf}$ (i.e., during the time of axon out- 

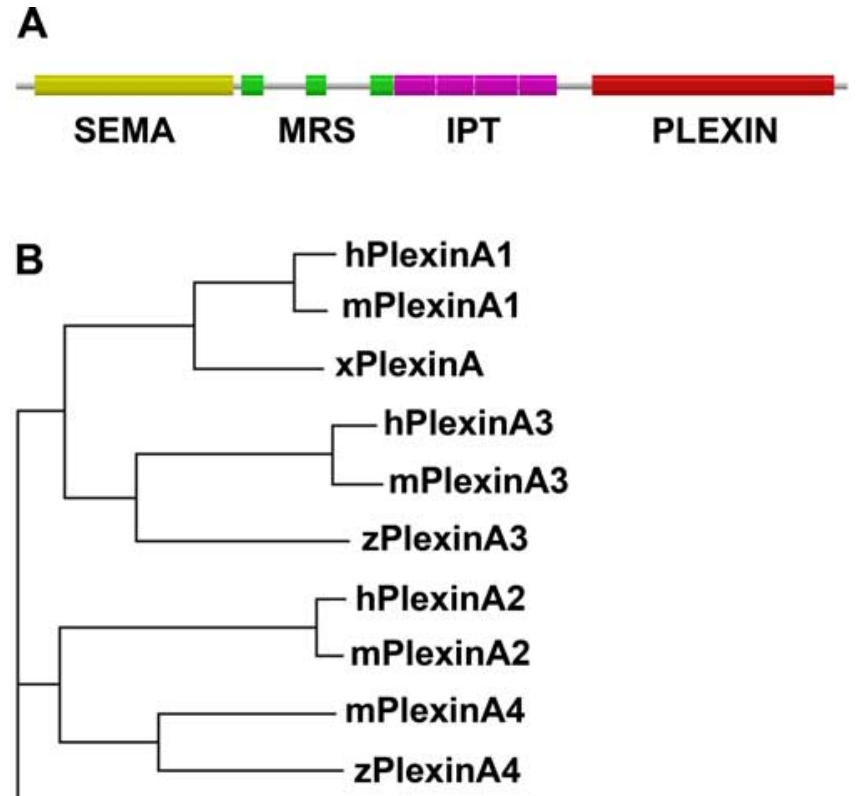

0.1

Figure 1. Structural features and identity of plexinA3 in zebrafish. $A$, Domain structure of plexinA3. SEMA, Semaphorin domain; PLEXIN, plexin domain; MRS, Met-related sequence. $\boldsymbol{B}$, Multiple comparisons in a phylogenetic tree group zebrafish plexinA 3 with plexinA 3 homologs in other vertebrates. Drosophila plexinA was added as an outgroup. The scale bar represents 10 substitutions per 100 aa. $z$, Zebrafish; $m$, mouse; $h$, human; $x$, Xenopus; d, Drosophila.

growth of primary motor neurons) (Fig. $2 A, B$ ). Axons and somata of motor neurons are labeled by GFP in an hb9:GFP transgenic fish (Flanagan-Steet et al., 2005). In situ hybridization of the motor neuron markers islet- 1 and islet- 2 in these transgenic fish confirmed GFP expression in primary motor neurons (data not shown). Double labeling of plexinA3 mRNA with GFP immunohistochemistry in hb9:GFP transgenic embryos at $24 \mathrm{hpf}$ revealed colocalization of the mRNA in GFP-positive motor neuron clusters from which the $\mathrm{CaP}$ axon had just started to grow in developmentally younger caudal segments. Conspicuous plexinA3 mRNA expression was also found in more dorsal GFP-negative spinal neurons (Fig. 2C-E). In more rostral segments in which the MiP axon could be seen to grow out, the mRNA was detectable in adjacent cells that are likely the $\mathrm{CaP}$ and MiP primary motor neurons (Fig. $2 \mathrm{~F}-\mathrm{H}$ ). The extra-spinal pathway of motor axons did not express detectable levels of plexinA3 mRNA. Thus, plexinA3 mRNA is expressed in primary motor neurons during axon outgrowth.

\section{Reduction of plexin $\mathrm{A} 3$ expression leads to aberrant branching} and multiple exits of motor nerves

Two translation-blocking plexinA3 morpholinos of nonoverlapping sequence significantly reduced expression of a coinjected myc-tagged plexinA3 mRNA that included the binding sites for both morpholinos. This was determined in immunohistochemistry for the myc epitope (data not shown). Thus, both plexinA3 morpholinos efficiently bind to their target sequence in vivo.

Ventral motor nerve growth in plexinA3 morpholinoinjected embryos was analyzed at $24 \mathrm{hpf}$ using anti-tubulin immunohistochemistry (Fig. $2 I-N$ ). Injection of $1 \mathrm{~mm}$ plexinA3 morpholinol led to abnormal growth of primary motor axons. Aberrations of ventral motor nerves, which normally grow as one unbranched nerve beyond the ventral edge of the notochord at 24 hpf (Fig. 2I,L), can be grouped into two categories: hemisegments that showed an additional nerve exiting the spinal cord (Fig. $2 M, N$ ) or nerves that were abnormally branched (Fig. $2 J, K)$.

In $64 \%$ of the affected hemisegments, mostly one additional nerve of variable length grew ventrally from an additional exit point in the ventral spinal cord (Fig. $2 M, N$ ). The additional nerve ran parallel to the main nerve or joined it at variable positions dorsal of the horizontal myoseptum. In $68 \%$ of the hemisegments showing additional exit points, it could not be resolved whether the nerve emanated rostral or caudal to the segment border because the nerves grew very close to it. In the remaining hemisegments, $73 \%$ of the additional exit points were located in the posterior half of the somites and 25\% were in the anterior half of the somites or in both the anterior and posterior somite half $(2 \%)$. On average, $4.7 \pm 0.4$ hemisegments per embryo had multiple exits in affected embryos.

Ventral motor nerves were aberrantly branched in $35 \%$ of the affected hemisegments (Fig. $2 J, K$ ). The vast majority of these branches $(82 \%)$ were directed caudally. Bifurcated (10\%), rostrally (5\%), and bilaterally (3\%) branched nerves were observed less frequently. On average, $3.4 \pm 0.2$ hemisegments per embryo showed aberrant branching in affected embryos.

The effects were dose dependent with 26,43 , and $64 \%$ of the embryos showing aberrant nerve branching and 18,56 , and $94 \%$ of the embryos showing additional exit points from the spinal cord after injections of $0.25,0.5$, and $1 \mathrm{~mm}$ morpholino1, respectively. Injecting $1 \mathrm{~mm}$ morpholino2 phenocopied these effects ( $83 \%$ of embryos were affected by abnormal branching; $95 \%$ of embryos were affected by additional exits). Injections of $1 \mathrm{~mm}$ of a morpholino in which five bases were mismatched $(5 \mathrm{~mm}$ morpholino) had no effect ( $14 \%$ of embryos were affected by branching; $12 \%$ of embryos were affected by additional exits) (for statistical significance, see Table 1). Thus, knock-down of plexinA3 induces both branching of ventral motor nerves and additional exit points from the spinal cord, preferentially in the posterior half of the trunk segments.

To determine whether dorsal motor axons, which are obscured in anti-tubulin-labeled embryos, were affected by the morpholino treatment, we analyzed hb9:GFP transgenic fish at $31 \mathrm{hpf}$. At this time point, GFP-positive axons had grown into the dorsal MiP pathway at the level of the yolk extension in uninjected animals (Fig. 2O). In $1 \mathrm{~mm}$ plexinA3 morpholino1- $(n=$ 10 embryos) or morpholino2- $(n=13$ embryos $)$ injected hb9: GFP embryos, axons were also present in the MiP pathway, including the segments with multiple exits ( $n=47$ segments). Interestingly, in nine of these segments, the additional exit points of ventral motor axons also produced additional axons that grew dorsally (Fig. 2P). Most of these dorsally growing axons were located more laterally than the normal MiP axons as determined from confocal image stacks (data not shown). This indicates that these ectopic axons did not simply follow an MiP pathway. Branching away from the normal MiP pathway was also slightly increased by morpholino treatment (Fig. $2 P$ ). The frequency of dorsal motor nerves that were branched ventral to the level of GFP-positive ventral spinal neurons was $33.4 \pm 2.84 \%$ hemisegments per embryo ( $n=327$ hemisegments) in morpholinotreated animals and $12.1 \pm 2.04 \%$ hemisegments per embryo $(n=215$ hemisegments; Mann-Whitney $U$ test; $p<0.0001)$ in hb9:GFP embryos injected with $5 \mathrm{~mm}$ morpholino ( $n=14 \mathrm{em}-$ bryos). Thus, additional nerves and increased nerve branching occur in both ventral and dorsal primary motor axon paths. 
A
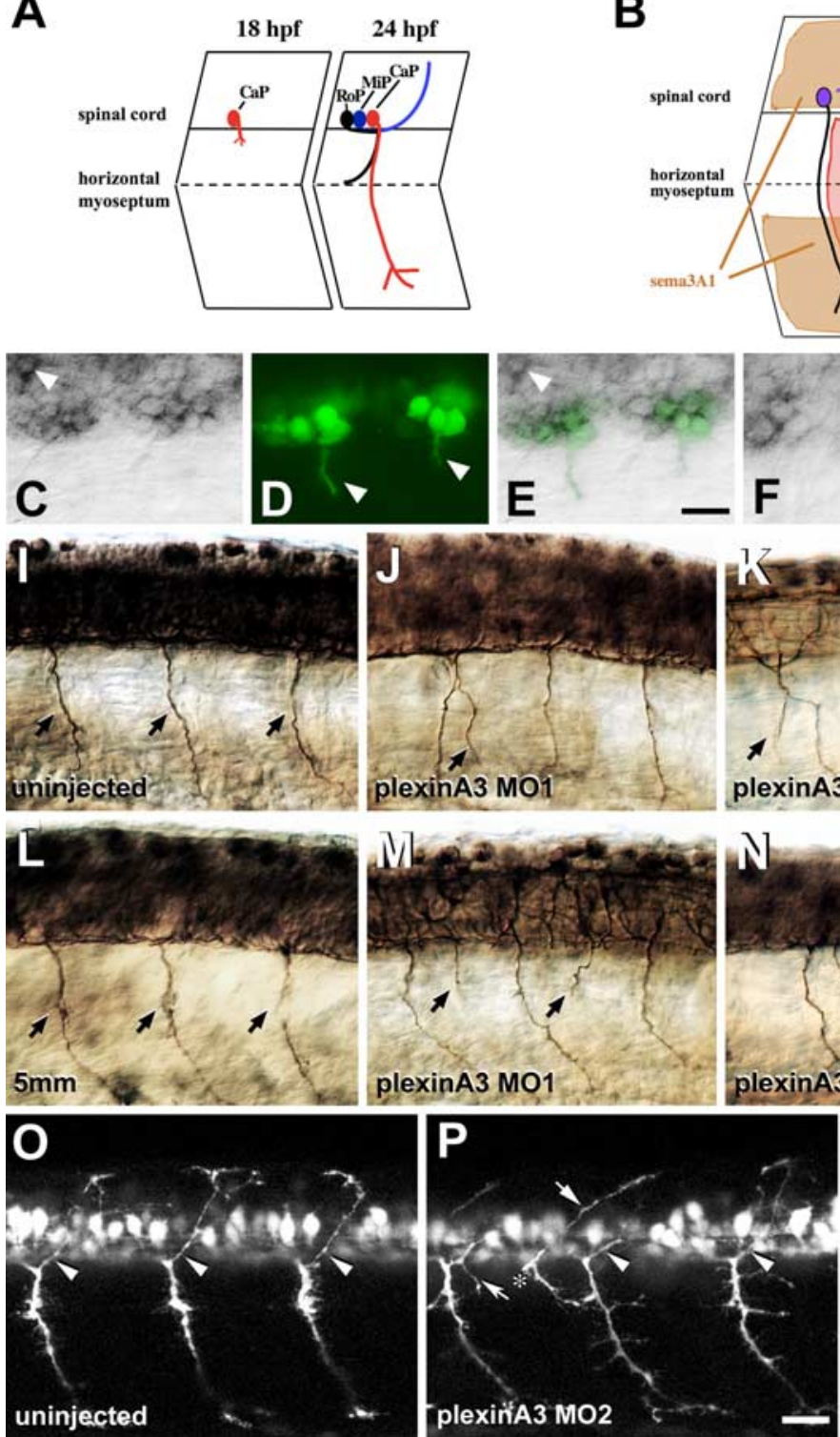

Figure 2. Expression and function of plexinA3 in primary motor neurons. $A, B, A$ schematic representation of the outgrowth of primary motor axons, which is preceded by the $\mathrm{CaP}_{\mathrm{P}}$ axon at $\sim 18 \mathrm{hpf}$, is given in a lateral view of one trunk segment in $\boldsymbol{A}$. $\boldsymbol{B}$, The distribution of semaphorin ligands relative to the ventral motor axon pathway. $\mathbf{C}-\boldsymbol{H}$, In situ hybridization of plexinA 3 mRNA shows expression in clusters of GFP-immunopositive motor neurons of hb9:GFP transgenic fish in lateral views at $24 \mathrm{hpf}$. C-E, A caudal region in which CaP axons ( $\boldsymbol{D}$, arrowheads) are just growing out. The arrowhead in $\boldsymbol{C}$ and $\boldsymbol{E}$ depicts a more dorsal, GFPimmunonegative cell that shows strong expression of plexinA3 mRNA. At higher magnification in $\boldsymbol{F}-\boldsymbol{H}$, two adjacent intensely plexinA3 mRNA-positive neurons in a more rostral segment are depicted. These are likely the CaP (right cell) and MiP (left cell) motor neurons, judging by the trajectories of the GFP-positive MiP (arrow) and CaP (arrowhead) axons. I-N, Lateral views at midtrunk levels of anti-tubulin-labeled whole-mounted $24 \mathrm{hpf}$ embryos are shown. In uninjected embryos (I) or those injected with $1 \mathrm{~mm}$ plexinA3, $5 \mathrm{~mm}$ morpholino $(5 \mathrm{~mm} ; \boldsymbol{L})$, single unbranched motor nerves (arrows in $\boldsymbol{I}$ and $\boldsymbol{L}$ ) grow ventrally out of the spinal cord. Injection of 1 mм plexinA3 morpholino1 (M01) induces branching (arrow in J) or a second spinal exit point for motor nerves per hemisegment (arrows indicate additional nerves in $\boldsymbol{M}$ ). Injection of $1 \mathrm{~mm}$ plexinA3 morpholino2 (M02) also induced aberrant branching ( $\boldsymbol{K}$, arrows) of the ventral motor nerve and additional nerves exiting the spinal cord ( $\boldsymbol{N}$, arrows). $\mathbf{0}, \boldsymbol{P}, A x o n s$ in the dorsal MiP pathway are visualized in hb9:GFP transgenic fish in selected confocal image stacks at 31 hpf, indicating normal growth in uninjected embryos ( $\mathbf{0}$, arrowheads), and excessive branching (curved arrow in $\boldsymbol{P}$ ) and supernumerary nerves (straight arrow in $\boldsymbol{P}$ ) in $1 \mathrm{~mm}$ plexinA3 morpholino2-injected embryos. The asterisk in $\boldsymbol{P}$ indicates an additional nerve exit point with a dorsal and ventral nerve branch exiting the spinal cord. Arrowheads in $\boldsymbol{P}$ point to normal appearance of axons in the dorsal motor axon pathway. Rostral is left in $\boldsymbol{A}$ to $\boldsymbol{P}$. Scale bars (in $\boldsymbol{E}$ ) $\boldsymbol{C}-\boldsymbol{E}, 25 \mu \mathrm{m}$; (in $\boldsymbol{H}) \boldsymbol{F}-\boldsymbol{H}, 12.5 \mu \mathrm{m}$; (in $\boldsymbol{N}$ ) $\boldsymbol{I}-\boldsymbol{N}, 25 \mu \mathrm{m}$; (in $\boldsymbol{P}$ ) $\mathbf{0}, \boldsymbol{P}, 25 \mu \mathrm{m}$.

Several other axonal structures labeled in anti-tubulin immunohistochemistry, such as commissures and the dorsoventral diencephalic tract in the head, as well as the posterior lateral line nerve and sensory Rohon-Beard axons, appeared unaffected by
plexinA3 knock-down (data not shown). The trigeminal ganglion, which shows defasciculation in its ophthalmic branch in plexinA3 deficient mice (Cheng et al., 2001; Yaron et al., 2005), appeared normal and contained $15.3 \pm 0.61$ ( $1 \mathrm{~mm}$ morpholino1) and $15.7 \pm 0.64$ ( $1 \mathrm{~mm}$ morpholino2) primary axon branches in morpholino-treated animals, which was not significantly different from embryos injected with $5 \mathrm{~mm}$ morpholino (16.4 \pm $0.65 ; p>0.1$ ) (supplemental Fig. $2 C-E$, available at www.jneurosci.org as supplemental material).

\section{Morpholino phenotypes are rescued by}

RNA overexpression and are not

because of alterations of the trunk environment

Overexpression of a full-length myctagged plexinA3 mRNA had no effect on motor axon growth as determined by antitubulin immunohistochemistry at $24 \mathrm{hpf}$ (data not shown). However, coinjection of plexinA3 morpholino2 (titrated to 0.3 $\mathrm{mm}$ ) with mRNA that does not have a binding sequence for the morpholino, led to a strong and significant reduction in the frequency of both abnormal branching (13\% affected embryos) and additional exits ( $16 \%$ affected embryos). This was compared with injection of $0.3 \mathrm{~mm}$ plexinA3 morpholino 2 alone at $24 \mathrm{hpf}$ (embryos affected by branching: $87 \%, p<0.0001 ; \mathrm{em}-$ bryos affected by additional exits: 49\%, $p<0.01$ ) (Table 1).

Analysis of markers of the horizontal and vertical myosepta, as well as spinal floorplate, motor neuron somata, commissural primary ascending interneurons in the spinal cord, and Mauthner neurons with their spinal axons, indicated normal differentiation of these structures after injection of $1 \mathrm{~mm}$ plexinA3 morpholino1 (supplemental Fig. 3, available at www. jneurosci.org as supplemental material).

\section{Genetic interactions of plexin $\mathrm{A} 3$ with sema $3 \mathrm{~A} 1$ and sema $3 \mathrm{~A} 2$}

To show synergisms of plexinA3 with potential ligands and coreceptors, we performed pairwise coinjections of morpholinos at subthreshold concentrations that did not elicit a phenotype in singleinjection experiments. Coinjections of sema3A1 (2 mM) and sema3A2 (2 mM) morpholinos with plexinA3 morpholino1 $(0.1 \mathrm{~mm})$ at subthreshold concentrations induced significant branching (sema3A1, $60 \%$ affected embryos; sema3A2, 34\% affected embryos) and additional exits (sema3A1, 38\% affected embryos; sema3A2, 52\% affected embryos) compared with embryos coinjected with 0.1 $\mathrm{mm}$ plexinA $3 \mathrm{~mm}$ morpholino and $2 \mathrm{~mm}$ sema3A1 morpholino 
(20\% embryos with aberrantly branched ventral motor nerves and 19\% embryos with additional exits of ventral motor nerves) (Fig. 3). Coinjections of morpholinos against the potential coreceptor NRP1a (0.1 mM) and against plexinA3 (0.1 $\mathrm{mm}$ ) at subthreshold concentrations (Fig. 3) (Feldner et al., 2005) were ineffective. These experiments suggest the possibility that plexinA3 is part of a receptor complex for sema3A1 and sema3A2. Differences in the magnitude of synergistic effects that are strongest for branching when sema3A1 morpholinos are coinjected with plexinA3 morpholinos or multiple exits when sema3A2 morpholinos are used suggest specific roles of the two sema3A paralogs.

\section{Discussion}

Relatively little is known about the contribution of the plexin coreceptors to the guidance of pioneer axons by semaphorins in vivo. Here, we identify plexinA3 as a key player for correct spinal exit of primary motor axons and unbranched growth of primary nerves in the trunk of zebrafish. Double knock-down experiments suggest a complex interplay of ligands in the trunk environment and receptor components in primary motor neurons.

PlexinA3 expression in motor neurons is pivotal for motor axon growth. Up to $95 \%$ of plexinA3 morpholino-injected embryos showed specific types of aberrations, and 30\% of all hemisegments analyzed were aberrant. This effect is larger than that found in comparable studies of other proteins in motor axon growth (Feldner et al., 2005; Sato-Maeda et al., 2006). Two sequence-independent morpholinos yielded identical results, and all of the phenotypes were almost completely rescued by supplementing plexinA 3 by overexpression. Using various markers, we could not find detectable changes in other axons or the spinal cord and trunk structures of morpholino-treated embryos. This suggests a major and specific function of plexinA3 in primary motor neurons.

PlexinA3 in dorsal and ventral motor axons may be necessary to correctly integrate repellent cues from semaphorins during axon outgrowth. The receptor knock-down phenotypes observed showed additional exits from the spinal cord and branching of the ventral and dorsal motor nerve that are consistent with a release of axon growth from environmental restrictions. Indeed, class 3 semaphorins are expressed in the trunk environment (Fig. $2 B$ ) and are thought to signal through plexin receptors. Synergistic effects in double morpholino injections of plexinA3 with sema3A homologs support a role for plexinA3 as a signaltransducing receptor component for repellent sema3A signals. Also consistent with such an interaction, knock-down of sema3A1 alone induces similar phenotypes to plexinA3 knockdown, including aberrant branching. However, shortened axons were also observed to a lesser extent (Sato-Maeda et al., 2006). Conversely, overexpression of sema3A1 or sema3A2 mainly induces reduced growth of motor axons (Roos et al., 1999; Halloran et al., 2000). In mammals, plexinA3 also mediates semaphorininduced pruning of axonal branches (Bagri et al., 2003). However, pruning appears not to be prominent during primary motor axon differentiation (Liu and Westerfield, 1990).

We provide evidence for subtle differences in the function of the two zebrafish homologs of sema3A for ventral motor nerve growth. Coinjections of plexinA3 and sema3A1 morpholinos pri-

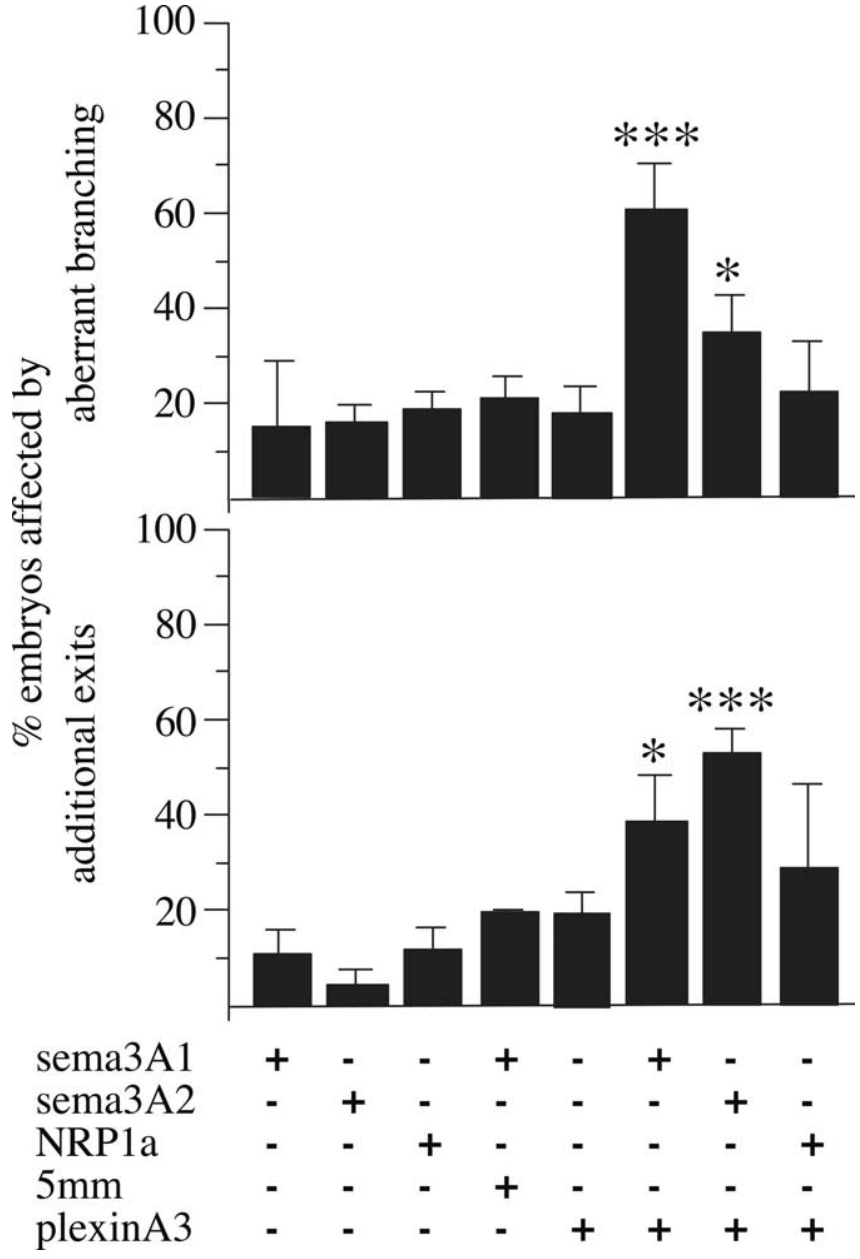

Figure 3. Combining plexinA 3 with semaphorin morpholinos has synergistic effects. $5 \mathrm{~mm}$, Control morpholino based on plexin morpholino 1 with five mismatched bases. For concentrations, see Results. ${ }^{*} p<0.05 ;{ }^{* * *} p<0.001$.

marily induced nerve branching, whereas coinjections of plexinA3 and sema3A2 morpholinos primarily induced additional nerve exits. This may be explained by differential distribution of the ligands (schematically shown in Fig. 2 B). Sema3A mRNA is only expressed in the dorsal and ventral myotome, leaving a corridor free of sema3A1 mRNA expression that includes the ventral edge of the spinal cord. Sema3A1 may therefore be more important during ventral growth when axons have ex- 
ited the spinal cord (Sato-Maeda et al., 2006). In contrast, sema3A2 mRNA is expressed continuously along the dorsoventral axis of the somite, including the level of nerve exit from the spinal cord but only in the caudal part of the somite (Roos et al., 1999). Thus, sema3A2 could restrict additional spinal exit points of motor nerves in the caudal part of the somite. Under plexinA3 knock-down conditions, consequently, $73 \%$ of the additional exits that were not exactly at the border between two segments occurred in the caudal half of the somite. Moreover, most aberrant nerve branches (82\%) were also directed caudally. This bias may indicate a loss of sensitivity to a repellent sema3A2 activity in the caudal half of the somite under plexinA3 knock-down conditions. However, it is still unknown what keeps nerves from branching rostrally and from exiting the spinal cord in the rostral half of the somite (Bernhardt et al., 1998).

Interestingly, combined injections of morpholinos to NRP1a and plexinA3 did not produce synergistic effects, although single knock-down of plexinA3 and NRP1a did induce partially overlapping phenotypes (nerve branching, additional exits). It is possible that efficiency/concentrations of morpholinos were not suitable to reveal such a possible interaction. However plexinA3 appears to preferentially associate with NRP2 in mammals (Cheng et al., 2001). A homolog of NRP2, NRP2b, is also expressed in primary motor neurons in zebrafish (gene expression database: http:// zfin.org/cgi-bin/webdriver?MIval=aa-xpatselect.apg). Moreover, there is at least one additional class 3 semaphorin expressed in the trunk of zebrafish, sema3G, which could influence motor axon growth (Stevens and Halloran, 2005). PlexinA1, which has not yet been cloned in zebrafish, may also play a role in the motor neuron system. Interestingly, the requirement for plexinA3 in specific axons in zebrafish appears to differ from that in mice: a plexinA3-deficient mouse shows fasciculation defects of trigeminal axons but apparently normal motor axon growth (Cheng et al., 2001; Yaron et al., 2005), whereas in zebrafish, motor axons but not trigeminal axons are affected by plexinA3 knock-down.

The anatomical simplicity of the primary motor system in zebrafish makes it an excellent tool to unravel the in vivo interactions of several ligands and their receptors that determine the outgrowth of pioneer axons. It will, for example, be interesting to find out whether mutations in the semaphorin signaling cascade will be discovered in genetic screens (Birely et al., 2005; GulatiLeekha and Goldman, 2006). We conclude that growth and path finding of primary motor axons in zebrafish is governed by a complex interplay of different semaphorin ligands and receptors of which plexinA3 is a crucial component. Forward and reverse genetic approaches have the potential to elucidate path finding of pioneer axons in this in vivo model in unprecedented detail.

\section{References}

Bagri A, Cheng HJ, Yaron A, Pleasure SJ, Tessier-Lavigne M (2003) Stereotyped pruning of long hippocampal axon branches triggered by retraction inducers of the semaphorin family. Cell 113:285-299.

Beattie CE (2000) Control of motor axon guidance in the zebrafish embryo. Brain Res Bull 53:489-500.

Becker T, Becker CG, Schachner M, Bernhardt RR (2001) Antibody to the HNK-1 glycoepitope affects fasciculation and axonal pathfinding in the developing posterior lateral line nerve of embryonic zebrafish. Mech Dev 109:37-49.

Becker T, Ostendorff HP, Bossenz M, Schlüter A, Becker CG, Peirano RI, Bach I (2002) Multiple functions of LIM domain-binding CLIM/NLI/ Ldb cofactors during zebrafish development. Mech Dev 117:73-83.

Bernhardt RR, Schachner M (2000) Chondroitin sulfates affect the formation of the segmental motor nerves in zebrafish embryos. Dev Biol 221:206-219.

Bernhardt RR, Goerlinger S, Roos M, Schachner M (1998) Anterior- posterior subdivision of the somite in embryonic zebrafish: implications for motor axon guidance. Dev Dyn 213:334-347.

Birely J, Schneider VA, Santana E, Dosch R, Wagner DS, Mullins MC, Granato M (2005) Genetic screens for genes controlling motor nervemuscle development and interactions. Dev Biol 280:162-176.

Cheng HJ, Bagri A, Yaron A, Stein E, Pleasure SJ, Tessier-Lavigne M (2001) Plexin-A3 mediates semaphorin signaling and regulates the development of hippocampal axonal projections. Neuron 32:249-263.

Chenna R, Sugawara H, Koike T, Lopez R, Gibson TJ, Higgins DG, Thompson JD (2003) Multiple sequence alignment with the Clustal series of programs. Nucleic Acids Res 31:3497-3500.

Feldner J, Becker T, Goishi K, Schweitzer J, Lee P, Schachner M, Klagsbrun M, Becker CG (2005) Neuropilin-1a is involved in trunk motor axon outgrowth in embryonic zebrafish. Dev Dyn 234:535-549.

Flanagan-Steet H, Fox MA, Meyer D, Sanes JR (2005) Neuromuscular synapses can form in vivo by incorporation of initially aneural postsynaptic specializations. Development 132:4471-4481.

Giger RJ, Cloutier JF, Sahay A, Prinjha RK, Levengood DV, Moore SE, Pickering S, Simmons D, Rastan S, Walsh FS, Kolodkin AL, Ginty DD, Geppert M (2000) Neuropilin-2 is required in vivo for selective axon guidance responses to secreted semaphorins. Neuron 25:29-41.

Gulati-Leekha A, Goldman D (2006) A reporter-assisted mutagenesis screen using alpha 1-tubulin-GFP transgenic zebrafish uncovers missteps during neuronal development and axonogenesis. Dev Biol 296:29-47.

Halloran MC, Sato-Maeda M, Warren JT, Su F, Lele Z, Krone PH, Kuwada JY, Shoji W (2000) Laser-induced gene expression in specific cells of transgenic zebrafish. Development 127:1953-1960.

Huber AB, Kania A, Tran TS, Gu C, De Marco Garcia N, Lieberam I, Johnson D, Jessell TM, Ginty DD, Kolodkin AL (2005) Distinct roles for secreted semaphorin signaling in spinal motor axon guidance. Neuron 48:949-964.

Kameyama T, Murakami Y, Suto F, Kawakami A, Takagi S, Hirata T, Fujisawa $\mathrm{H}$ (1996) Identification of plexin family molecules in mice. Biochem Biophys Res Commun 226:396-402.

Kimmel CB, Ballard WW, Kimmel SR, Ullmann B, Schilling TF (1995) Stages of embryonic development of the zebrafish. Dev Dyn 203:253-310.

Kruger RP, Aurandt J, Guan KL (2005) Semaphorins command cells to move. Nat Rev Mol Cell Biol 6:789-800.

Lee P, Goishi K, Davidson AJ, Mannix R, Zon L, Klagsbrun M (2002) Neuropilin-1 is required for vascular development and is a mediator of VEGF-dependent angiogenesis in zebrafish. Proc Natl Acad Sci USA 99:10470-10475.

Liu DW, Westerfield M (1990) The formation of terminal fields in the absence of competitive interactions among primary motoneurons in the zebrafish. J Neurosci 10:3947-3959.

Maestrini E, Tamagnone L, Longati P, Cremona O, Gulisano M, Bione S, Tamanini F, Neel BG, Toniolo D, Comoglio PM (1996) A family of transmembrane proteins with homology to the MET-hepatocyte growth factor receptor. Proc Natl Acad Sci USA 93:674-678.

Miyashita T, Yeo SY, Hirate Y, Segawa H, Wada H, Little MH, Yamada T, Takahashi N, Okamoto H (2004) PlexinA4 is necessary as a downstream target of Islet2 to mediate Slit signaling for promotion of sensory axon branching. Development 131:3705-3715.

Patel NH, Martin-Blanco E, Coleman KG, Poole SJ, Ellis MC, Kornberg TB, Goodman CS (1989) Expression of engrailed proteins in arthropods, annelids, and chordates. Cell 58:955-968.

Roos M, Schachner M, Bernhardt RR (1999) Zebrafish semaphorin Z1b inhibits growing motor axons in vivo. Mech Dev 87:103-117.

Sato-Maeda M, Tawarayama H, Obinata M, Kuwada JY, Shoji W (2006) Sema3al guides spinal motor axons in a cell- and stage-specific manner in zebrafish. Development 133:937-947.

Schweitzer J, Becker T, Lefebvre J, Granato M, Schachner M, Becker CG (2005) Tenascin-C is involved in motor axon outgrowth in the trunk of developing zebrafish. Dev Dyn 234:550-566.

Stevens CB, Halloran MC (2005) Developmental expression of sema3G, a novel zebrafish semaphorin. Gene Expr Patterns 5:647-653.

Tongiorgi E (1999) Tenascin-C expression in the trunk of wild-type, cyclops and floating head zebrafish embryos. Brain Res Bull 48:79-88.

Yaron A, Huang PH, Cheng HJ, Tessier-Lavigne M (2005) Differential requirement for Plexin-A3 and -A4 in mediating responses of sensory and sympathetic neurons to distinct class 3 Semaphorins. Neuron 45:513523. 